\title{
Confusion noise level due to galactic and extragalactic binaries
}

\author{
Peter L Bender and Dieter Hils \\ JILA, University of Colorado, CB440, Boulder, CO 80309 , USA
}

Received 11 November 1996, in final form 28 February 1997

\begin{abstract}
We have revised our earlier rough estimate of the combined galactic and extragalactic binary confusion noise level curve for gravitational waves. This was done to correct some numerical errors and to allow for roughly three frequency bins worth of information about weaker sources being lost for each galactic binary signal that is removed from the data. The results are still based on the spectral amplitude estimates for different types of galactic binaries reported by Hils et al in 1990, and assume that the gravitational wave power spectral densities for other galaxies are proportional to the optical luminosities. The estimated confusion noise level drops to the LISA instrumental noise level at between roughly 3 and $8 \mathrm{mHz}$.
\end{abstract}

PACS numbers: $0480 \mathrm{~N}, 0430$

\section{Introduction and summary}

Estimates of the abundance of a number of types of galactic binaries and of the resulting expected spectral amplitudes of their gravitational wave signals were published in 1990 by Hils et al [1]. (For earlier work by various authors, see references in [1].) But, the number of galactic binaries per frequency resolution bin is also important. At sufficiently low frequencies, well below $1 \mathrm{mHz}$, there will be so many binaries on average in even a 1 cycle/yr frequency bin that very few bins will be empty and large number statistics will prevail. However, at substantially higher frequencies and neglecting complications due to Doppler shifts, enough bins will contain no galactic binaries so that information about signals from weaker extragalactic sources can be obtained.

For the frequency range from $1-10 \mathrm{mHz}$ of particular interest in this paper, the decrease in the number of binaries per bin with increasing frequency is mainly due to the rapid increase in the rate of energy loss from gravitational radiation. This leads to the number of binaries per bin decreasing as the $-\frac{11}{3}$ power of the frequency over a substantial frequency range. The transition region where a significant number of bins begin to be open and substantially higher sensitivity to extragalactic signals can be achieved is of particular interest for the Laser Interferometer Space Antenna (LISA) mission.

Previously, we made an extremely crude estimate of what the confusion noise limit would be for LISA $[2,3]$, based on the gravitational wave spectral amplitudes given by Hils et al [1]. In the absence of direct observations, one assumption made was that the actual space density of close white dwarf binaries (CWDBs) is $10 \%$ of the theoretical value based on the calculations of Webbink [4]. A possibly equally uncertain assumption was that all galaxies out to large redshifts have the same average ratio of gravitational wave spectral amplitude to luminosity as our galaxy. In addition, some theoretical and numerical errors 
were made, and the Doppler shifts and the amplitude distribution of galactic binary signals were ignored. The results were used to give some idea of the effective sensitivity of the LISA mission as a function of frequency $[2,3]$.

In the present paper, we correct the known errors in the previous results and describe the procedures we have used. However, the main approximations are still made. The results will be given in figure 2, both for $10 \%$ and $100 \%$ of the space density of CWDBs derived from the results of Webbink [4].

For the future, it has become clear that some allowance for an additional class of galactic binaries needs to be made. This class can be described roughly as helium cataclysmic variables (see e.g. $[5,6]$ ). An important subclass is the AM CVn stars, also called interacting white dwarf binaries (IWDBs), which have been discussed as gravitational wave sources by Warner [7] and Hellings [8]. They are likely to have a substantial effect on the confusion noise limit for LISA at frequencies of roughly $1-3 \mathrm{mHz}$. Despite uncertainties in the progenitor population and in the evolutionary history of such binaries, some allowance needs to be made for their effect.

\section{General approach}

The spectral amplitudes for a number of different types of galactic binaries are shown in figure 1 . The values at intervals of 0.2 in the $\log$ of the frequency were taken from Hils et al [1], interpolated and somewhat smoothed. The values for the $\mathrm{He}-\mathrm{He}, \mathrm{He}-\mathrm{CO}$ and $\mathrm{CO}-\mathrm{CO}$ CWDBs were recalculated to give separate curves, since the different signal strengths for these types of sources will be of interest in future studies. These CWDB curves are for

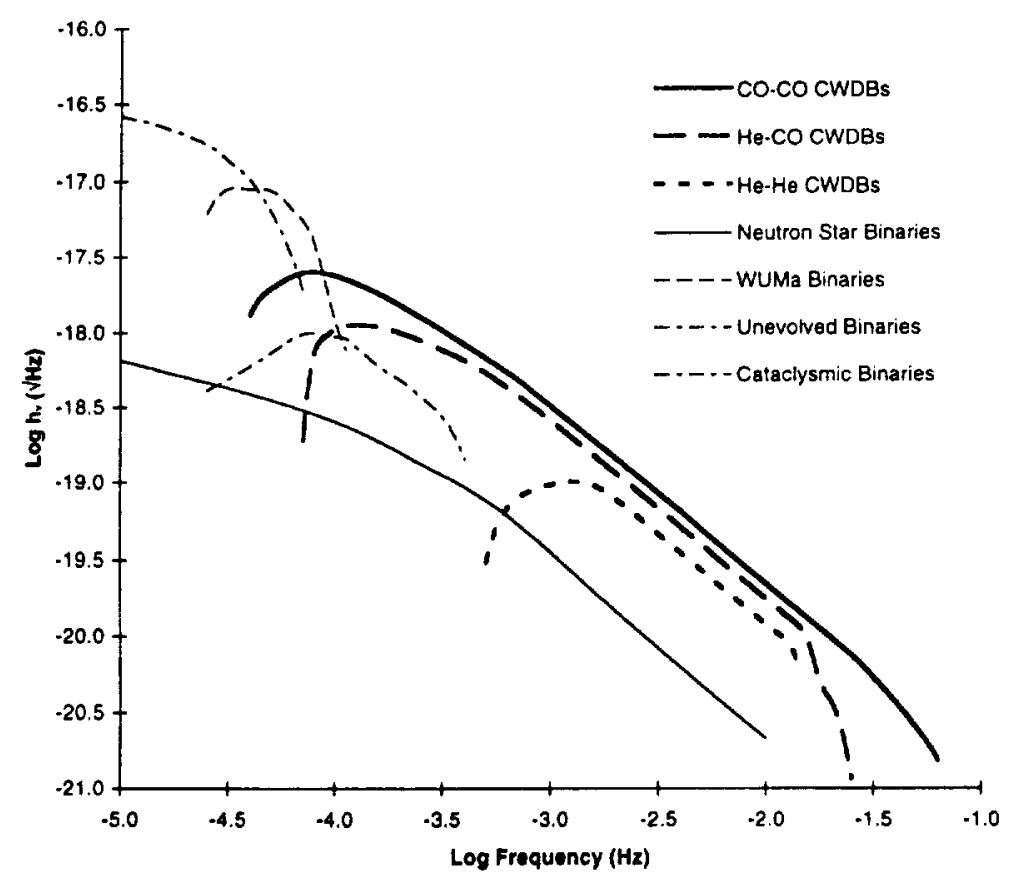

Figure 1. Root-mean-square estimates of the signal levels due to different types of galactic binaries. The three CWDB curves are for $10 \%$ of the theoretical space density derived from the results of Webbink [4]. 
$10 \%$ of the theoretical space densities found from the results of Webbink [4].

The frequency range from $1 \mathrm{mHz}$ to 3 or $4 \mathrm{mHz}$ is of particular interest, since this is where the transition from being limited mainly by galactic binaries to being limited by extragalactic binaries takes place. We assume that all sources will be solved for if they have an apparent signal strength greater than two to three times the noise level remaining after their effects are removed from the data set, even though a higher $S / N$ ratio is usually needed to have a high confidence level that the signals are real. The appropriate threshold would be set by simulations.

For extragalactic binaries, equal thickness spherical shells make equal contributions to the spectral amplitude for moderate redshifts. At larger distances, the effect of the redshift becomes important. In the future, models for binary abundance variations during galactic evolution will need to be considered. However, for now we neglect such effects and integrate all the way out to $z=5$, which may somewhat compensate for evolution effects. The results are insensitive to the cut-off value chosen. The estimated galactic luminosities given by Efstathiou et al [9] were used. The resulting extragalactic spectral amplitude was found to be about a factor of 10 lower than the galactic spectral amplitude over the frequency range of interest.

\section{Combination of galactic and extragalactic confusion noise estimates}

An important question is how to combine the effects of galactic and extragalactic confusion noise. We start from the very rough concept of some bins being filled by galactic binaries and some empty. In reality, sidebands are put on the signal from each galactic binary because of the phase and amplitude modulation due to motion and orientation change of the antenna during the year. Examples for $1 \mathrm{mHz}$ frequency and two source directions are given in section 3.7 of [3], where roughly 20 or 30 sidebands are shown. For higher frequencies, the number of sidebands will be higher. The sidebands produced for amplitude modulation only have been described by Giampieri [10].

In the transition region, information about weak extragalactic sources will have to be obtained from residuals after sets of phase-related sidebands due to a number of individual galactic binaries have been fitted to the data. However, since the number of parameters determining the motional sidebands is small, we expect that the information lost will not be substantially more than if only a few frequency bins were affected per binary.

In our earlier confusion noise estimate [2,3], we assumed that one bin of information about weaker extragalactic sources would be lost for each galactic binary. We pictured the data set as being broken into two parts, with one consisting of bins with no galactic source and the other containing the rest of the bins. Then, we treated the $S / N$ ratio as being the sum of the contributions from these two data sets. However, it was pointed out to us by Hellings [8] that more than one bin's worth of information at the extragalactic confusion noise level would be lost for each galactic binary solved for.

We now assume that roughly three bins of information at the extragalactic confusion noise level are lost for each galactic binary solved for. This is based on there being a phase and amplitude for each source polarization to be solved for, as well as two source direction coordinates, while each bin has only two parameters that can be solved for. This approximation is a crude one, and certainly will need to be checked by simulations, but we do not expect it to be a major source of error in the results.

Near frequency $f$, let the probability of no galactic binary in a given 1 cycle/yr frequency bin be $C(0)$. If $r$ is the mean number of galactic binaries per frequency bin, $C(0)=\exp (-r)$. The fraction of bins not substantially affected by solving for galactic binaries is thus assumed 
to be $\exp (-3 r)$. Next, let $N$ (gal) and $N($ ext $)$ be the RMS confusion noise levels for the galactic and extragalactic binaries separately. Then, the contribution to $(S / N)^{2}$ from a potentially interesting extragalactic source of strength $h$, using only information from the unaffected bins, is given roughly by the following:

$$
\left[\left(S^{2}\right) /\left(N^{2}\right)\right]_{a}=[\exp (-3 r)]\left\{\left(h^{2}\right) /\left[N(\operatorname{ext})^{2}\right]\right\} .
$$

Similarly, for the fraction of bins that are affected:

$\left[\left(S^{2}\right) /\left(N^{2}\right)\right]_{b}=\{1-\exp (-3 r)\}\left[\left(h^{2}\right) /\left\{\left[N(\mathrm{gal}, \bmod )^{2}\right]+\left[N(\mathrm{ext})^{2}\right]\right\}\right]$.

Here $N$ (gal, mod) is the RMS value of the confusion noise level due to galactic binaries, modified by being averaged over only the fraction of bins that are affected by solving for galactic binaries. Thus

$$
N(\text { gal, mod })^{2}=\left\{\left[N(\mathrm{gal})^{2}\right] /[1-\exp (-3 r)] .\right.
$$

Finally, combining these contributions to $(S / N)^{2}$ quadratically, the effective combined confusion noise level $N$ (eff) is estimated to be given approximately by the following:

$$
\begin{aligned}
1 /\left[N(\mathrm{eff})^{2}\right]= & {[\exp (-3 r)] /\left[N(\mathrm{ext})^{2}\right] } \\
& +[1-\exp (-3 r)] /\left\{\left[N(\mathrm{gal})^{2}\right] /[1-\exp (-3 r)]+\left[N(\text { ext })^{2}\right]\right\} .
\end{aligned}
$$

The results are given in figure 2 . The full curve is the standard expected LISA sensitivity curve $[2,3]$ for a $S / N$ ratio of 5 and 1 yr of observations. The broken curve is the effective confusion noise estimate from equation (4), also for $S / N=5$ and 1 year of observations, using $10 \%$ of the theoretical CWDB spatial density obtained from the results of Webbink [4]. The dotted curve is the same, but for $100 \%$ of the CWDB density.

The curve for the $10 \% \mathrm{CWDB}$ density results has its region of rapid decrease moved to higher frequencies than our earlier estimate by roughly a factor of two $[2,3]$. This is mainly due to a numerical error in the previous work. However, about a third of the difference is due to our now assuming that more than one bin of information at the extragalactic confusion noise level is lost for each galactic binary solved for, as suggested by Hellings [8].

For the actual case of substantial phase and amplitude modulations due to motion and orientation change of the antenna, the problem is considerably more complex than our simple model. Simulations of the combined confusion noise level after solving for galactic binaries will be needed. However, the above method for estimating the results seems useful, until actual simulations have been carried out.

One other minor point concerns the treatment of instrumental noise. In principle, such noise should be combined with the extragalactic confusion noise before $N$ (eff) is calculated. However, we have kept it separate for convenience, and assume that the quadratic sum of the instrumental noise and the confusion noise estimate will be close to being correct.

\section{Discussion}

The information available concerning the abundance of noninteracting CWDBs was reviewed by Hils et al [1] in 1990. At that time, Robinson and Shafter [11] had carried out a search for CWDBs with orbital periods of $30 \mathrm{~s}$ to $3 \mathrm{~h}$, and had not found any. However, Saffer et al [12] had recently discovered one detached white dwarf binary with a period of 1.55 days. Thus, the existence of at least one noninteracting CWDB had been established.

More recently, there have been new observations of CWDBs. In 1990, Bragaglia et al [13] reported that WD0957-666 is a double degenerate with an apparent period of 1.15 days. 


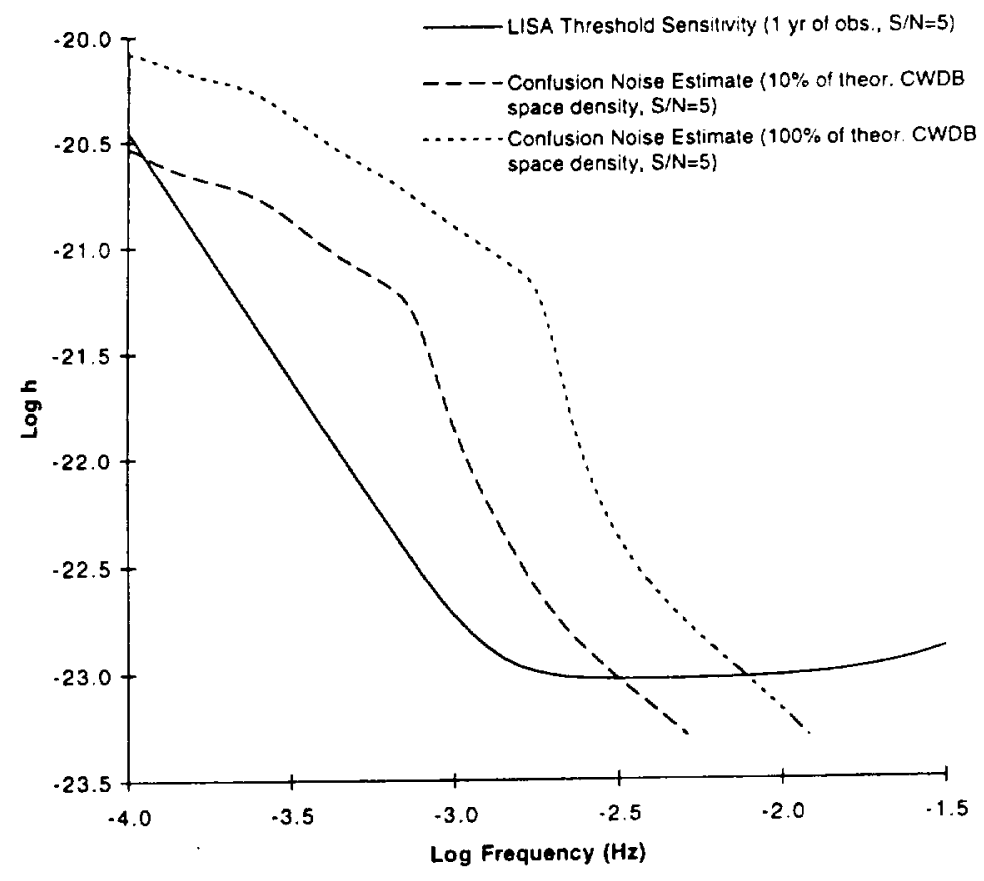

Figure 2. Confusion noise estimates for the combination of galactic and extragalactic binaries. The expected LISA threshold sensitivity is also shown. All curves correspond to 1 yr of observations and a $S / N$ ratio of 5 .

Then, in 1995, Marsh et al [14] reported discovering five detached close binaries out of seven observed white dwarfs chosen for having masses below $0.45 M_{\odot}$. Their observations support at least four of these being CWDBs. One had a period of roughly $4 \mathrm{~h}$. Also, Marsh [15] found an additional one with a period of $3.47 \mathrm{~h}$. Still more recently, Moran et al [16] have found that WD0957-666 actually has a period of $88 \mathrm{~min}$ rather than 1.15 days. As a result, there are now three CWDBs known with periods of about $4 \mathrm{~h}$ or less, and thus lifetimes before coalescence due to gravitational radiation of less than the Hubble time (see also Iben et al [17]).

Unfortunately, it has not yet been possible to convert the new observations into specific estimates of the CWDB abundance. The low-mass white dwarfs observed by March et al were chosen because it appeared unlikely theoretically that such stars with masses below $0.45 M_{\odot}$ could be produced by any mechanism other than common envelope evolution. Thus, finding a large fraction of close binaries was not surprising. The issue of selection effects in searches for CWDBs still needs to be addressed before a space density can be determined. For this reason, the confusion noise estimate based on $10 \%$ of the theoretical abundance derived from the results of Webbink [4] still seems to be a useful reference noise level for the LISA mission and is shown in figure 2. However, the higher estimate based on $100 \%$ of the theoretical abundance is also shown.

For the $10 \%$ of the theoretical abundance assumption, there will be thousands of individual CWDBs resolvable at frequencies above $3 \mathrm{mHz}$ and many more for $100 \%$ abundance. It should be remembered that helium cataclysmic variables still need to be included and that the extragalactic contributions to these curves have the additional uncertainty associated with a lack of knowledge of how typical the CWDB abundance in 
our galaxy is. If substantial numbers of CWDBs are not present in our galaxy, there still are expected to be thousands of helium cataclysmic variables resolvable between $1 \mathrm{mHz}$ and $3 \mathrm{mHz}$.

\section{Acknowledgments}

We thank C Cutler, R Hellings, T Marsh, R Webbink and members of the LISA Science Team for information and suggestions that have aided our work. The research described in this paper was carried out under support from the National Aeronautics and Space Administration under grant no NAGW-4865.

\section{References}

[1] Hils D, Bender P L and Webbink R F 1990 Astrophys. J. 360 75-94

[2] Danzmann K et al 1993 LISA-proposal for a laser-interferometric gravitational wave detector in space Report MPQ 177 Max-Planck-Institut für Quantenoptik, Garching, Germany

[3] Danzmann K et al 1996 LISA Pre-Phase A Report. Report MPQ 208 Max-Planck-Institut für Quantenoptik, Garching, Germany

[4] Webbink R F 1984 Astrophys. J. 277 355-60

[5] Savonije G J, de Kool M and Van Den Heuvel E P 1986 J. Astron. Astrophys. 155 51-7

[6] Iben I and Tutukov A V 1991 Astrophys. J. 370 615-29

[7] Wamer B 1995 Astrophys. Space Sci. 225 249-70

[8] Hellings R 1996 paper and comments presented at Int. Conf. on Gravitational Waves: Sources and Detectors (Cascina)

[9] Efstathiou G, Ellis R S and Peterson B A 1988 Mon. Not. R. Astron. Soc. 232 431-61

[10] Giampieri G 1995 Mon. Not. R. Astron. Soc. submitted

[11] Robinson E L and Shafter A W 1987 Astrophys. J. 322 296-301

[12] Saffer R A, Liebert J and Olszewski E W 1988 Astrophys. J. 334947

[13] Bragaglia A, Greggio L, Renzini A and D'Odorico S 1990 Astrophys. J. 365 L13-7

[14] Marsh T R, Dhillon V S and Duck S R 1995 Mon. Not. R. Astron. Soc. $275828-40$

[15] Marsh T R 1995 Mon. Not. R. Astron. Soc. 275 Ll-5

[16] Moran C K J, Marsh T R and Bragaglia A 1997 Mon. Not. R. Astron. Soc, in preparation

[17] Iben I, Tutukov A V and Yungelson L R 1997 Astrophys. J. 475 291-9 